\title{
Pilot Study of Nuclear Scintigraphy To Assess Cough Clearance in DMD
}

\author{
Daniel J. Weiner ${ }^{1}$, Hoda Abdel-Hamid ${ }^{2}$, and T. E. Corcoran ${ }^{3}$ \\ ${ }^{1}$ Children's Hospital of Pittsburgh \\ ${ }^{2}$ Children's Hospital of Pittsburgh of UPMC Department of Pediatrics \\ ${ }^{3}$ University of Pittsburgh
}

January 6, 2022

\section{Pilot Study of Nuclear Scintigraphy To Assess Cough Clearance in DMD}

To the Editor:

Respiratory muscle weakness as a consequence of neuromuscular disease results in ineffective cough, atelectasis, and pneumonia. Augmented airway clearance treatments such as mechanical in-exsufflation and high frequency chest compression have been shown to decrease morbidity and mortality in children with neuromuscular disease, although the evidence supporting these therapies in clearance of airway secretions is indirect.

We sought to pilot the use of nuclear imaging of the clearance of pulmonary secretions before and after voluntary coughing and airway clearance treatment to explore the role of measures of pulmonary function to predict airway clearance. This technique has been used for some time in patients with cystic fibrosis but has not been applied to patients with neuromuscular disease or to assess the utility of airway clearance devices. To this end, we recruited 7 outpatient subjects (7.8-21 years of age) with Duchenne Muscular Dystrophy when clinically well and categorized as "Early Ambulatory" (requiring minimal assistive devices, $\mathrm{n}=4$ ); "Early Non-ambulatory" (using wheelchair some of time, $\mathrm{n}=2$ ); and "Late Non-Ambulatory" (always using wheelchair, $\mathrm{n}=1$ ). This study was approved by local Institutional Review Board.

Spirometry was performed according to ATS specifications and normalized using Global Lung Initiative equations. Peak cough flow was measured from total lung capacity (TLC) and data was normalized with previously published equations[1]. Maximal inspiratory pressure (MIP) and maximal expiratory pressure (MEP) were measured at the mouth using a hand-held pressure manometer and maximal pressure from FRC was also be measured at the nares (SNIP) during a sniff maneuver[2] using a small nasal occluder and handheld transducer Maximal static pressures and SNIP pressures were normalized using previously published data.

For imaging of mucus clearance, $4 \mathrm{mCi}$ of Technetium [Tc-99m] sulfur-colloid particles in $2 \mathrm{ml}$ of normal saline were delivered by nebulizer using a defined breathing pattern for 2 minutes [3]. During continuous imaging, subjects were asked to voluntarily cough every 10 seconds for 1 minute using guidance from a metronome, and this sequence was repeated 3 times. The subject then received an airway clearance treatment for 30 minutes using a high frequency chest compression device with pressure adjusted between 80-100\% of maximum, and compression frequencies cycled between $5 \mathrm{~Hz}-15 \mathrm{~Hz}$. The device was paused every 10 minutes at which time the patient was asked to cough every 10 seconds over 1 minute.

Image analysis was performed according to previously published methods. Measurements of radioactivity in the right lung were corrected for background and radioactive decay and normalized by starting radioactive 
counts. A single retention curve was generated from the initial cough clearance period and the period during which the high frequency chest compression device was operated. Cough clearance (CC) represents the percent of total deposited radioactivity cleared during the associated period. We designated the percentage cleared by 18 voluntary coughs over 3 minutes as $\mathrm{CC}_{\mathrm{vol}}$, and the percentage cleared during three 10 min periods of $\mathrm{HFCC}$ and 18 voluntary coughs over 3 minutes as $\mathrm{CC}_{\mathrm{HFCC}} \cdot \mathrm{CC}_{\mathrm{vol}}$ and $\mathrm{CC}_{\mathrm{HFCC}}$ were correlated with physiologic measures including peak cough flow (PCF), MIP/MEP, and SNIP using Spearman's correlation.

Pulmonary function was overall well preserved (FVC $92 \% \pm 35 \%$ ). Respiratory muscle strength (in $\mathrm{cmH}_{2} \mathrm{O}$ ) decreased with disease severity (e.g. MIP EA $73.5 \pm 26$, ENA $62.5 \pm 14.8$, LNA 47). Peak cough flow (\% predicted) decreased with severity (EA $94 \pm 11$, ENA $79 \pm 9$, LNA 46). Complete details are provided in the online supplement.

Representative imaging of two subjects is shown (Figure 1). Significant intra-subject differences were seen in clearance with voluntary cough and with cough augmented with HFCC vest (Figure 2). Voluntary cough was responsible for $46-96 \%$ of the total clearance and HFCC-augmented clearance for 3-53\% of total clearance. For most patients, the kinetics of clearance with HFCC-augmented cough did not appear very different than for voluntary cough.

Statistical comparisons between disease stages were hampered by the small sample size in each group. Nonetheless, while there appeared to be a trend for decreasing $\mathrm{CC}_{\mathrm{vol}}$ with stage of disease (Figure 2), age, and PCF, we found no statistically significant correlations between voluntary cough clearance and measures of pulmonary function ( $\mathrm{FVC}, \mathrm{r}=0.39$ ), respiratory muscle strength ( $\mathrm{PCF}, \mathrm{r}=-0.63$; SNIP $\mathrm{r}=-0.12$, MIP $\mathrm{r}=0.43$, MEP $\mathrm{r}=0.29)$, age $(\mathrm{r}=-0.57)$, height $(\mathrm{r}=-0.49)$, weight $(\mathrm{r}=-0.37)$. Additionally, we did not find statistically significant correlations between augmented cough clearance and measures of pulmonary function $(\mathrm{FVC}, \mathrm{r}=0.40)$, respiratory muscle strength $(\mathrm{PCF}, \mathrm{r}=-0.08$; SNIP $\mathrm{r}=-.02$, MIP $\mathrm{r}=-0.07$, MEP $\mathrm{r}=0.05)$, age $(\mathrm{r}=-0.39)$, height $(\mathrm{r}=-0.75)$, weight $(\mathrm{r}=-0.03)$.

This study is the first we are aware of to directly examine cough clearance of secretions using nuclear scintigraphy in patients with DMD. Importantly, the measurements were well tolerated by subjects. We did not find a relationship between CPF, respiratory muscle strength, or spirometry and voluntary cough clearance. However, these results must be qualified based on the small number of enrolled subjects. We do note that many of the patients had good cough clearance. Additionally, HFCC did not consistently alter the rate of secretion clearance above noted with voluntary cough.

While Bach et al[4] showed that PCF $>160 \mathrm{~L} /$ min was a predictor of successful tracheal decannulation in adults with neuromuscular disease, Airen et al[5] found that the majority of healthy children $<13$ years old generated PCFs $<270 \mathrm{l} / \mathrm{min}$ despite having the ability to generate MEP $>60 \mathrm{cmH}_{2} \mathrm{O}$. This suggests that $\mathrm{PCF}$ may change with age and that an absolute threshold cannot be used across the age span. There are no studies specifically examining use of HFCC in patients with DMD. As patients with DMD are expected to have impairment in cough, mechanical in-exsufflation would be expected to assist with airway clearance by replacing the function of weak respiratory muscles in a way that HFCC does not. Lechtzin et al[6] showed that in adults with neuromuscular diseases, HFCC decreased inpatient costs and costs for treating pneumonia compared to the year prior to initiating HFCC. Measures of pulmonary function, including PCF, were not assessed. It would be of interest to examine secretion removal with in-exsufflation using this nuclear imaging method.

While we were not able to demonstrate a relationship between cough clearance and measures of respiratory muscle strength, peak cough flow, or FVC, it is possible that this relationship exists and would be demonstrated in a larger population. However, the longstanding assumption that PCF is a reliable indicator of need for airway clearance, especially in children, is not supported by these data. The biggest limitation of our pilot study is the small sample size. We suspect enrollment was limited due to preference for interventional studies. Another limitation is that for our population, the impairment of respiratory muscle strength was mild for most subjects. Additional subjects with more advanced weakness may have helped clarify the relationship between respiratory muscle strength and airway clearance. Finally, there can be day-to-day variations of 
clearance.

There are now a number of airway clearance technologies being proposed for patients with neuromuscular and airway diseases (e.g. cystic fibrosis, ciliary dyskinesia). These include high frequency chest compression vests, battery-powered vests, in-exsufflation devices (with or without oscillation), intrapulmonary percussive ventilation devices, and handheld oscillating positive expiratory pressure devices. We propose that the imaging methodology used in this study could be utilized to compare these different techniques, some of which are costly and with limited or no comparison data. In addition, there are patients that will be unable to cooperate with pulmonary function measurements and an imaging assessment of airway clearance may be useful to determine optimal strategies.

In conclusion, we demonstrated that use of nuclear scintigraphy is a feasible technique to examine airway clearance for patients with respiratory muscle weakness, as it has been used for patients with cystic fibrosis. Additional studies will be needed to further examine the relationship between pulmonary function measures and secretion removal.

Daniel J. Weiner $\mathrm{MD}^{1}$,

Hoda Abdel Hamid $\mathrm{MD}^{2}$,

Timothy E. Corcoran $\mathrm{PhD}^{3}$

Divisions of Pediatric Pulmonology ${ }^{1}$ and Neurology ${ }^{2}$, Department of Pediatrics

UPMC Children's Hospital of Pittsburgh

Division of Pulmonary, Allergy and Critical Care Medicine ${ }^{3}$, Department of Medicine

University of Pittsburgh School of Medicine

\section{References}

1. Bianchi, C. and P. Baiardi, Cough peak flows: standard values for children and adolescents. Am J Phys Med Rehabil, 2008.87 (6): p. 461-7.

2. Marcelino, A.A., et al., Reference values of sniff nasal inspiratory pressure in healthy children. Pediatr Pulmonol, 2020.55 (2): p. 496-502.

3. Zeman, K.L., et al., Comparison of 133 xenon ventilation equilibrium scan (XV) and 99m technetium transmission (TT) scan for use in regional lung analysis by $2 D$ gamma scintigraphy in healthy and cystic fibrosis lungs. J Aerosol Med Pulm Drug Deliv, 2013. 26 (2): p. 94-100.

4. Bach, J.R. and L.R. Saporito, Criteria for extubation and tracheostomy tube removal for patients with ventilatory failure. A different approach to weaning. Chest, 1996. 110 (6): p. 1566-71.

5. Airen, M., J. McDonough, and H. Panitch, Cough peak flow measurements in normal school-age children , in American Thoracic Society . 2004. p. 169.

6. Lechtzin, N., L.F. Wolfe, and K.D. Frick, The Impact of High-Frequency Chest Wall Oscillation on Healthcare Use in Patients with Neuromuscular Diseases. Ann Am Thorac Soc, 2016.13 (6): p. 904-9. 


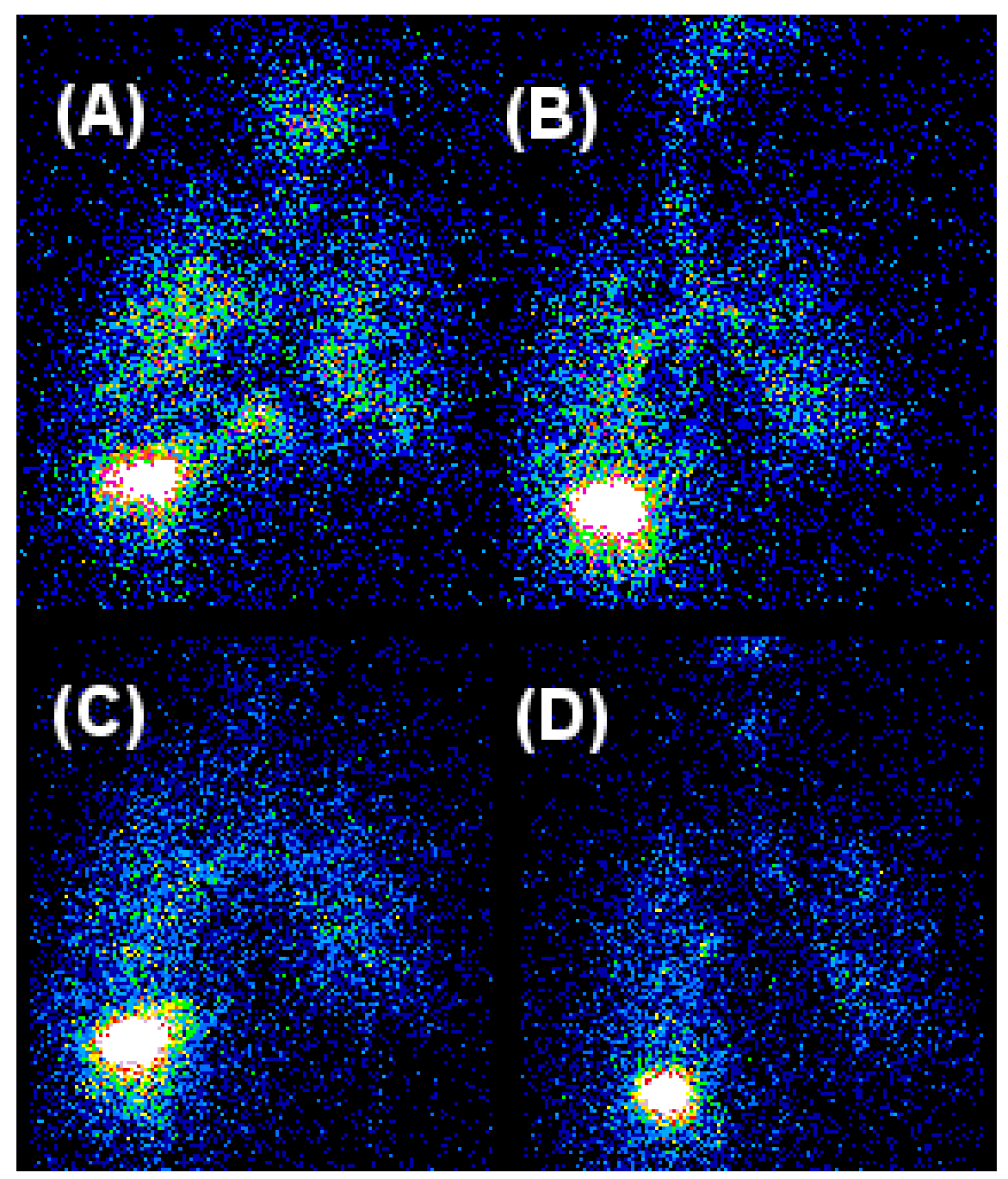




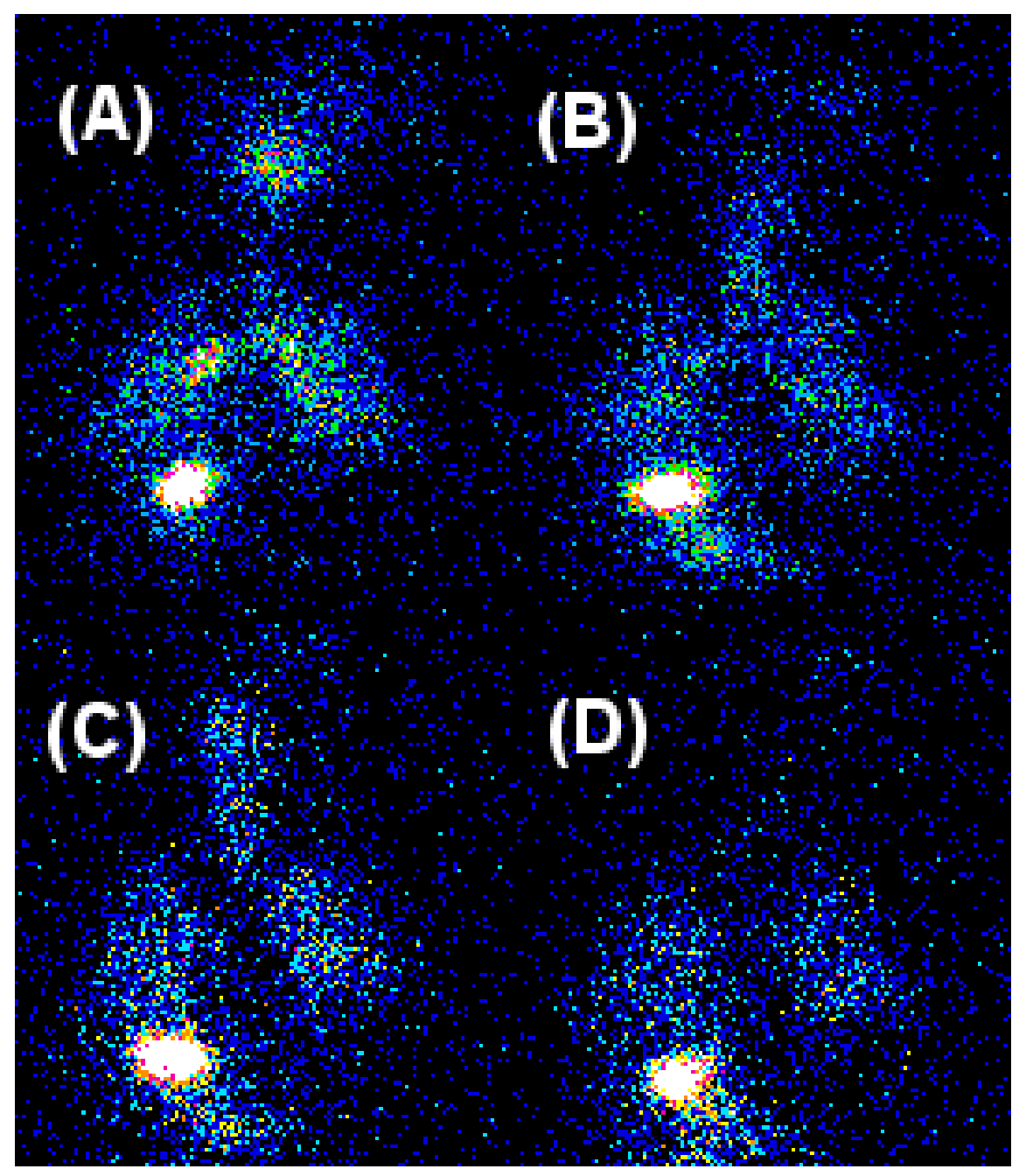

Figure 1, Left. Image montage from subject \#4 $\left(\mathrm{CC}_{\mathrm{vol}} 36 \%, \mathrm{CC}_{\mathrm{HFCC}} 5 \%\right)$. Figure 1, Right. Image montage from subject \#8 ( $\mathrm{CC}_{\mathrm{vol}}$ : 0\%, $\left.\mathrm{CC}_{\mathrm{HFCC}}: 25 \%\right)$. For both, (A) Pre-coughing, (B) After 18 coughs in 18 min (C) Prior to vest-augmented coughing (D) After 30 minutes vest treatment and 18 coughs over 40 minutes. Images are posterior planar images depicting Tc-SC deposited in the lungs. 


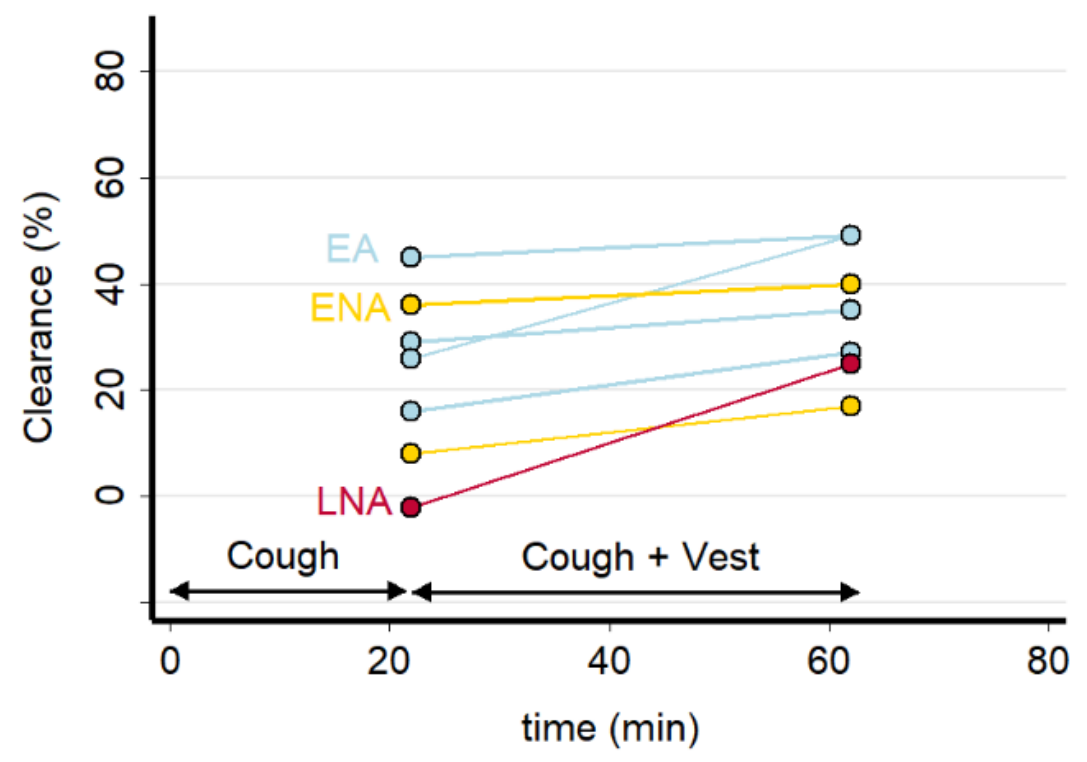

Figure 2. Clearance (\%) of radiotracer in the lung after voluntary cough, and after a 30 minute treatment with HFCC and intermittent voluntary coughing. 